\title{
Procrastinación: Una Revisión de su Medida y sus Correlatos
}

\section{Procrastination: A Review of Scales and Correlates}

\author{
Juan F. Díaz-Morales ${ }^{1}$
}

\begin{abstract}
Resumen
La procrastinación consiste en la tendencia generalizada a aplazar el inicio y/o finalización de tareas planificadas para ser realizadas en un tiempo determinado. Tal tendencia a la postergación suele acompañarse de malestar subjetivo y no sólo es una cuestión de baja responsabilidad y gestión del tiempo, sino que supone un verdadero problema de auto-regulación a nivel cognitivo, afectivo y conductual. En este artículo de revisión se proporciona una visión general de la investigación realizada sobre procrastinación en población española organizada en los siguientes apartados: marco conceptual de la procrastinación, instrumentos de medida, prevalencia y diferencias según edad, sexo y ocupación, modelos estructurales, tipos de procrastinación y correlatos (estilos de personalidad, perspectiva temporal, matutinidadvespertinidad).
\end{abstract}

Palabras clave: procrastinación, instrumentos de medida, prevalencia, personalidad, perspectiva temporal, matutinidad-vespertinidad

\begin{abstract}
Procrastination is the general tendency to postpone the start and/or end of tasks planned to be performed in a given time. Such a tendency to postpone is usually accompanied by subjective discomfort and not only is it a question of low responsibility and time management, but it is a real problem of self-regulation at the cognitive, affective and behavioral levels. In this review an overview of the research carried out on procrastination with Spanish people is provided. The review is organized in the following sections: conceptual framework of procrastination, measurement instruments, prevalence and differences according to age, sex and occupation, structural models, types of procrastination and correlates (personality styles, temporal perspective, morningness-eveningness).
\end{abstract}

Keywords: procrastination, scales, prevalence, personality styles, temporal perspective, morningnesseveningness

\footnotetext{
${ }^{1}$ Profesor Titular de Universidad (Psicología Diferencial). Coordinador del Máster Universitario Oficial en Género y Salud. Facultad de Psicología, Universidad Complutense de Madrid. Campus de Somosaguas, s/n, 28223, Pozuelo de Alarcón, Madrid, España. Correo: juanfcodiaz@psi.ucm.es
} 


\section{Introducción}

\section{Procrastinar es dejar habitualmente para mañana lo que puedes hacer hoy}

En la sociedad occidental las personas habitualmente se plantean metas a corto, medio y largo plazo relacionadas con uno mismo/a, la educación, el trabajo, la salud, etc. lo cual implica trazar un plan para conseguirlas, ya sea un plan explícito o implícito, y una estimación del tiempo necesario para realizarlo. Si las actividades que se planean realizar se llevan a cabo en tiempo, muy probablemente las consecuencias positivas de tales actividades sean evidentes. Por el contrario, si las conductas planeadas y orientadas a futuro no se realizan en el tiempo estimado, la probabilidad de que aparezcan consecuencias negativas se incrementa.

En general, las personas que "dejan para mañana lo que pueden hacer hoy" son vistas como personas perezosas, vagas o irresponsables, así valoradas incluso por otras personas con la misma tendencia. La falta de diligencia a la hora de realizar tareas planeadas en un tiempo estimado se asocia con cierto malestar subjetivo, y si tal tendencia se da en diferentes áreas vitales, entonces se trata de una persona que procrastina. La procrastinación consiste en la tendencia generalizada a aplazar el inicio y/o finalización de tareas planificadas para ser realizadas en un tiempo determinado. Tal tendencia a la postergación suele acompañarse de malestar subjetivo y no sólo es una cuestión de baja responsabilidad y gestión del tiempo, sino que supone un verdadero problema de auto-regulación a nivel cognitivo, afectivo y conductual.

En el ámbito académico universitario tal tendencia es muy común, dado que muchas de las actividades académicas deben realizarse en un plazo estipulado. No obstante, el/la estudiante que no entrega el trabajo de clase a tiempo, puede cumplir diligentemente otras tareas tales como sacar entradas para un concierto o quedar a la hora estipulada con amigos/a. En este caso, la tendencia a aplazar el inicio de la tarea escolar es denominada procrastinación académica, muy conocida en el ámbito educativo y muy poco abordada desde los departamentos de orientación universitarios. La procrastinación también ha sido considerada como un factor relevante en otros ámbitos tales como el ámbito de la salud (Sirois, 2015), el mundo laboral (Metin, Taris, \& Peeters, 2016), económico (Ericson, 2017) o familiar (Soysa \& Weiss, 2014), por poner algunos ejemplos. Para una revisión puede consultarse la obra de Ferrari (2010).

La procrastinación, así pues, es entendida como una demora en el inicio y/o finalización de una tarea o actividad que se tenía la intención de realizar, y que suele acompañarse de malestar, ansiedad y/o preocupación (Lay, 1986). Los estudios que han tratado de identificar las motivaciones de tal conducta concluyen que es necesario diferenciar entre conductas de postergación y de procrastinación (Ferrari, Johnson, \& McCown, 1995). Postergación consiste en aplazar la ejecución de una tarea con el fin de darle prioridad a otra más productiva en ese momento, lo cual no constituye un problema para la persona. Se aplazan o postergan unas actividades propositivamente y con el objetivo de realizar otra sin que esto suponga sensación de malestar psicológico para la persona. Sinónimos de postergar son aplazar, demorar, posponer o retrasar. Por el contrario, procrastinación designa un problema relacionado con la intención de realizar una tarea y una frecuente falta de diligencia ya sea para empezarla, desarrollarla o finalizarla. Este proceso generalmente se acompaña de sentimientos de inquietud y abatimiento y sus consecuencias sólo se observan a largo plazo. En conclusión, no todas personas que aplazan la realización de tareas, son procrastinadoras.

Aunque la procrastinación es una tendencia conductual bien conocida desde cientos de años, el estudio de las causas, manifestaciones, consecuencias, evaluación y tratamiento comenzó en la década de los ochenta (Lay, 1986). El primer libro que reunía las investigaciones hasta la fecha fue publicado en el año 1995 (Ferrari et al., 1995), posteriormente se publicó un libro sobre procrastinación académica (Schouwenburg, Lay, Pychyl, \& Ferrari, 2004), y posteriormente han aparecido diversas obras sobre la temática. Como se ha señalado anteriormente, una buena síntesis divulgativa sobre los ámbitos a los que se está aplicando la procrastinación puede encontrarse en el libro del profesor Ferrari (2010). Así, la investigación científica sobre la procrastinación se 
ha ido incrementando progresivamente, siendo un tema cada vez más interesante en el ámbito educativo, laboral, clínico y social.

No obstante, en este artículo de revisión se proporciona una visión general de la investigación realizada sobre procrastinación en población española. No se pretende realizar una revisión sistemática ni exhaustiva de la investigación realizada sobre procrastinación a nivel internacional, sino de la investigación desarrollada en población de habla española. Esta revisión está motivada por las frecuentes consultas sobre las escalas de medida de la procrastinación en español, dado que el primer estudio psicométrico de las escalas de procrastinación en población española fue publicado en inglés. Buena parte de tal investigación ha sido realizada en el seno del Grupo de Investigación de la Universidad Complutense de Madrid, Estilos de Personalidad, Género y Salud (EPSY, https://www.ucm.es/epsy/) durante la última década. La mayor parte de la investigación se ha realizado en población española, aunque también con población peruana, polaca y estadounidense, en las que se ha testado diferentes modelos de medida, o bien, se ha estimado la prevalencia de la procrastinación. Esta línea de investigación comenzó con el análisis psicométrico de las principales medidas de evaluación de la procrastinación en población española (Díaz-Morales, Ferrari, Díaz, \& Argumedo, 2006) y peruana (Argumedo, Díaz, Calderón, Díaz-Morales, \& Ferrari, 2005). Recientemente, se ha analizado también en población polaca (Blachnio, Przepiorka, \& DíazMorales, 2016). Estos estudios han resucitado el interés por la medida de la procrastinación puesto que hasta el año 2005 pocos investigadores/as abordaron empíricamente las siguientes cuestiones: ¿es la procrastinación un concepto unidimensional?, ¿pueden diferenciarse diferentes dimensiones?, ¿las medidas al uso permiten identificar diferentes formas de procrastinar?.

Posteriormente, se analizó la prevalencia de la procrastinación en países como Estados Unidos, Gran Bretaña, Australia, Venezuela, Perú y España (Ferrari, Díaz-Morales, O'Callaghan, Díaz, \& Argumedo, 2007), así como las diferencias de edad y sexo en población adulta española (Díaz-Morales, Ferrari, Díaz, \& Argumedo, 2006b). Buena parte de la investigación previa se había realizado en población universitaria, en donde no aparecían diferencias de edad debido a que la edad de la población universitaria está comprendida entre los 18 y $\operatorname{los} 25 / 30$ años (efecto de restricción de rango). Como la mayor parte de los estudios se han realizado en población adulta española no universitaria, se pudo verificar que las personas mayores procrastinan menos que las personas jóvenes. Tal tendencia fue replicada posteriormente en un estudio epidemiológico realizado en una muy amplia población británica (Steel \& Ferrari, 2013).

Además se han realizado estudios analizando el estilo de personalidad de las personas procrastinadoras en población adulta española (Díaz-Morales, Ferrari, \& Cohen, 2008) y peruana (Díaz, Argumedo, \& Díaz-Morales, 2009), así como con el autoconcepto (Ferrari \& DíazMorales, 2007) y el self (Ferrari, Driscoll, \& Díaz-Morales, 2007).

La definición de procrastinación incluye, necesariamente, dos elementos centrales: el malestar psicológico fruto del aplazamiento y la marco temporal. En este sentido, se ha estudiado la relación de la procrastinación con dos tipos de variables temporales: la perspectiva temporal (Ferrari \& Díaz-Morales, 2007) y la tipología circadiana (Díaz-Morales, Ferrari, \& Cohen, 2008). Por definición, las personas que procrastinan están muy poco orientadas al futuro y muy orientadas al presente $y$, por otra parte, las personas vespertinas, es probable que, en un contexto social matutino, tiendan a aplazar sus tareas hasta su mejor momento, la tarde-noche. Resulta interesante cómo distintas variables temporales puede que se relacionen con distintos tipos de procrastinación: la procrastinación por indecisión se relaciona con la falta de balance cognitivo de la orientación al pasado, presente y futuro; y la procrastinación conductual se relaciona con la preferencia vespertina por la tarde-noche.

Finalmente, el uso problemático de las redes sociales (por ejemplo, Facebook) se asocia con la procrastinación (Przepiorka, Błachnio, \& DíazMorales, 2016), lo cual reflejaría la falta de autoregulación de las personas procrastinadoras, quienes se embarcan con más frecuencia en tareas gratificantes a corto plazo demorando, 
posiblemente, las tareas que tienen que acometer en un tiempo determinado.

Obviamente, la investigación sobre la procrastinación es relativamente escasa comparada con otros ámbitos de la psicología. Y aunque más investigación es necesaria, por supuesto, en este artículo de revisión se presenta un resumen general de lo realizado hasta la fecha en población de habla española, con la confianza que estos logros susciten el interés del lector/a, así como de otros grupos de investigación e investigadores/as, y pueda darse continuidad a la investigación aquí presentada.

\section{¿Existe la procrastinación?}

El término procrastinación proviene del latín procrastinare, que literalmente significa "dejar para mañana" y se ha definido como la tendencia a aplazar el inicio y/o finalización de actividades que deben hacerse en un plazo de tiempo determinado, generando en la persona cierto malestar subjetivo (Ferrari et al., 1995).

Solomon y Rothblum (1984) sugieren que debido a que las definiciones de procrastinación acentúan tanto el retraso conductual como la ansiedad, ambos elementos de la definición deberían considerarse juntos. Desde este punto de vista, la procrastinación se ha definido como una tendencia irracional a retrasar las tareas que deberían completarse (Lay, 1986), como la demora innecesaria de actividades que finalmente se pretende completar, especialmente cuando se demora hasta el punto de crear malestar emocional. Schouwenburg et al. (2004) sugirieron que la procrastinación se infiere de las manifestaciones conductuales (por ejemplo la falta de puntualidad en las citas o la demora en la entrega de trabajos académicos) así como, a un nivel más cognitivo, de las intenciones de realizar actividades. Por último, Steel (2007) considera que la procrastinación consiste en "retrasar voluntariamente un curso de acción previsto a pesar de las consecuencias negativas que ello acarrea".

Para el procrastinador/a, el retraso temporal parece lógico y justificable; sin embargo, desde un punto de vista externo es irracional y puede dañar la percepción que los demás tienen de la persona. Para Lay (1986) la procrastinación es una función de la brecha entre la intención y la conducta: los/as procrastinadores/as no desarrollan conductualmente su intención de realizar la tarea. A veces, el procrastinador/a genera toda una serie de sesgos cognitivos generando excusas, infravaloraciones, remordimientos, así como acciones de auto-sabotaje con el fin de no realizar la conducta. Lay (1986) se basa en la teoría del comportamiento planificado (Ajzen, 1991) para remarcar que las actitudes no predicen suficientemente bien lo que hacen las personas, pero sí predicen la intención de realizar tales conductas. La intención conductual sí es un mejor predictor de comportamiento.

Las personas que se ven a sí mismas como procrastinadoras a menudo desean reducir tal tendencia estableciendo objetivos realistas y plazos para completar las tareas en un marco de tiempo razonable, pero generalmente siempre se subestima el tiempo necesario para llevarlas a cabo (Ariely \& Wertenbroch, 2002), sobreestimándose también los estados motivacionales futuros para realizar la tarea (Uzun, Ferrari, \& LeBlanc, 2018).

Además, diversos estudios han indicado que la procrastinación se relaciona con una variedad de variables de personalidad, incluyendo baja autoconfianza y autoestima (Ferrari \& DíazMorales, 2007b) y elevadas tendencias a la depresión, ansiedad social, impulsividad disfuncional, rigidez conductual y falta de energía (Ferrari \& Pychyl, 2000). La procrastinación se ha relacionado con dos rasgos principales de la personalidad, alto neuroticismo y baja responsabilidad, específicamente baja autodisciplina (Schouwenberg \& Lay, 1995). Así, en un primer intento teórico por articular una red de constructos relacionados con la procrastinación, Schouwenburg et al. (2004) consideraron la procrastinación como un concepto relacionado con rasgos como débil control de los impulsos, falta de persistencia, falta de disciplina en el trabajo, falta de habilidad para manejar el tiempo e incapacidad para trabajar metódicamente (ver también Díaz-Morales, Ferrari, Díaz \& Argumedo, 2006a).

Una de las cuestiones que ha surgido a raíz de estos estudios es la aparición de conceptos "novedosos" tales como procrastinación "activa" o "racional" (Chun Chu \& Choi, 2005). Tales términos carecen de base teórica, puesto que la 
procrastinación en sí misma implica un acto que la persona desea evitar, que genera malestar y que repercute negativamente en su vida cotidiana. No parece acertado denominar "procrastinación activa" a la mera postergación de tareas que no conlleven malestar para la persona. Para denominar tal acción hablamos de aplazar, demora o postergar. Tal y como proponen los propios autores de tal propuesta, la procrastinación estaría constituida por cuatro subdimensiones tales como la preferencia afectiva por la presión temporal, la decisión cognitiva de procrastinar, la capacidad conductual de cumplir fechas límites y la habilidad para lograr resultados satisfactorios (Choi \& Moran, 2009). Tales subdimensiones necesitan todavía ser validadas, no sólo psicométricamente, sino también teóricamente.

La procrastinación es un fenómeno complejo que integra los aspectos cognitivos, emocionales y conductuales, mas el intervalo temporal necesario para realizar la tarea (Ferrari, 2010). Tal complejidad se refleja en el influyente modelo propuesto por Steel (2007), quien plantea que la procrastinación se refleja en la ecuación $\mathrm{E} \times \mathrm{V} /$ $\Gamma \mathrm{D}$, donde $\mathrm{E}=$ expectativa o probabilidad de que ocurra un resultado, $\mathrm{V}=$ valor o deseabilidad de un resultado, $\Gamma=$ sensibilidad a los retrasos, y $\mathrm{D}=$ el tiempo hasta que esté disponible un estímulo gratificante. De acuerdo con esta función, la demora temporal que caracteriza a la procrastinación, es una función en la que se minimiza el valor de la mayor recompensa a largo plazo y se maximiza las menores recompensas a corto plazo (véase Steel, Svartdal, Thundiyil, \& Brothen, 2018).

\section{¿Cómo se mide?}

Entre los estudiantes, el indicador de procrastinación académica más utilizado ha sido el retraso en la entrega de trabajos con fecha establecida: los estudiantes que obtuvieron mayores niveles de procrastinación en las escalas de procrastinación general fueron más propensos a posponer la presentación de las tareas (Digdon \& Howell, 2008). Durante la década de 1990, se realizaron varios estudios cuasi-experimentales en situaciones reales en los que se validaron algunas de las escalas de evaluación más utilizadas (Ferrari et al., 1995). Por ejemplo, en el lugar de trabajo, los empleados se enfrentaron a tareas en las que probablemente fracasarían o tareas con fechas límites indefinidas o en la época navideña se estudió la tendencia comprar los regalos en el último momento. Tales estudios realizados en situaciones reales fueron recopilados en el excelente meta-análisis de Van Eerde (2003).

Tras la validación externa de las escalas de medida de la procrastinación, comenzaron a estudiarse sus propiedades psicométricas. En la actualidad, se han identificado varias medidas fiables y válidas de procrastinación. Entre los

Tabla 1. Inventario de Procrastinación para Adultos (Adult Inventory of Procrastination, AIP; McCown, Johnson, \& Petzel, 1989)

\begin{tabular}{|c|c|}
\hline \multicolumn{2}{|l|}{ Ítens } \\
\hline 1r. Pago mis facturas a tiempo. & 12345 \\
\hline 2r. Soy puntual para la mayoría de mis compromisos. & 12345 \\
\hline 3r. Preparo mi ropa la noche anterior a una entrevista para que no se me haga tarde. & 12345 \\
\hline 4. Me doy cuenta que suelo llegar más tarde de lo que quisiera a mis compromisos. & 12345 \\
\hline 5. No tengo las cosas hechas a tiempo. & 12345 \\
\hline 6. Si alguien estuviera impartiendo un curso sobre cómo tener las cosas hechas a tiempo, yo asistiría. & 12345 \\
\hline 7. Mis amigos y mi familia piensan que siempre espero hasta el último minuto. & 12345 \\
\hline 8r. Tengo las cosas importantes hechas con tiempo de sobra. & 12345 \\
\hline 9. No soy muy bueno en las reuniones con fecha establecida. & 12345 \\
\hline 10. Me encuentro corriendo cuando ya no queda tiempo. & 12345 \\
\hline 11r. Tengo las citas con el médico cuando debo, no me demoro en hacerlo. & 12345 \\
\hline 12r. Soy más puntual que la mayoría de la gente que conozco. & 12345 \\
\hline $\begin{array}{l}\text { 13r. Hago un mantenimiento rutinario de las cosas que poseo tan frecuentemente como debería hacerlo } \\
\text { (ej. cambiar el aceite al coche). }\end{array}$ & 12345 \\
\hline 14. Cuando tengo que estar en un lugar a cierta hora, mis amigos esperan que llegue tarde. & 12345 \\
\hline 15. En el último año, retrasar las cosas hasta el último minuto me ha costado dinero. & 12345 \\
\hline
\end{tabular}


Tabla 2. Escala de Indecisión (Decisional Procrastination, DP; Mann, 1982)

\begin{tabular}{l} 
Ítens \\
\hline 1. Pierdo bastante tiempo en detalles sin importancia antes de tomar la decisión final. \\
2. Incluso después de haber tomado una decisión, me demoro en llevarla a cabo. \\
3. No tomo decisiones hasta que me veo obligado(a) a hacerlo. \\
4. Retraso tanto mis decisiones que cuando me decido, ya es demasiado tarde. \\
5. Pospongo tomar decisiones. \\
Nota. Para calcular su puntuación en la escala tiene que sumar sus respuestas: epd1,epd2,epd3,epd4,epd5
\end{tabular}

adultos, las escalas más utilizadas han sido la escala de Procrastinación general (General Procrastination, GP; Lay, 1986), el Inventario de Procrastinación para adultos (Adult Inventory of Procrastination, AIP; McCown, Johnson, \& Petzel, 1989) o la escala de indecisión (Decisional Procrastination, DP; Mann, 1982). Entre los estudiantes, se ha utilizado con frecuencia la Escala de Evaluación de la Procrastinación Estudiante (Solomon \& Rothblum, 1984), el Inventario de Procrastinación de Aitken (Aitken Procrastination Inventory, API, Aitken, 1982) y la Escala de Procrastinación de Tuckman (Tuckman Procrastination Scale; Tuckman, 1991).

En el Inventario de Procrastinación para adultos (Adult Inventory of Procrastination, AIP; McCown, Johnson, \& Petzel, 1989), debe valorarse si cada afirmación describe lo que sucede habitualmente considerando una escala ordinal tipo likert desde 1 (no me describe en absoluto) hasta 5 (me describe totalmente). $\mathrm{Su}$ fiabilidad (consistencia interna) ha sido corroborada en diversos estudios con un alpha de Cronbach en torno a .83 (Díaz-Morales et al., 2006).

En población adulta española se extrajeron dos factores o componentes, denominados como falta de puntualidad y falta de planificación (DíazMorales et al., 2006a). En una muestra de adultos de Turquía se denominaron como aspectos positivos de evitación y aspectos negativos de evitación (Ferrari, Özer, \& Demir, 2009), mientras que en población italiana, la escala se consideró unidimensional (Mariani \& Ferrari, 2012). La puntuación promedio en población española oscila alrededor de un valor de 34 . Una persona que obtenga puntuaciones por debajo de 25 es muy poco procrastinadora con respecto a la población española, mientras que una persona que obtenga puntuaciones por encima de 43 tiende a ser muy procrastinadora (ver Tabla 1).

La procrastinación por indecisión, se ha evaluado mediante la Escala de Indecisión (Decisional Procrastination, DP; Mann, 1982), y del mismo modo que el anterior, debe valorarse si cada afirmación describe lo que sucede habitualmente considerando una escala ordinal tipo likert desde 1 (no me describe en absoluto) hasta 5 (me describe totalmente). Su fiabilidad (consistencia interna) ha sido corroborada en diversos estudios con un alpha de Cronbach en torno a .81 (Díaz-Morales et al., 2006).

En población adulta española el valor promedio de la Escala de Indecisión es 13. Una persona que obtenga una puntuación por debajo de 9 es poco indecisa, y por encima de 17 tiende a ser más indecisa que la población de referencia (ver Tabla 2).

Por último, la Escala de Procrastinación General (General Procrastination, GP; Lay, 1986) está compuesta por veinte preguntas. En diversos estudios previos ha sido corroborada su fiabilidad (consistencia interna) con un alpha de Cronbach en torno a .84 (Díaz-Morales et al., 2006).

El valor promedio de la Escala de Procrastinación General (GP) es de 51. Una puntuación por debajo de 40 indica una baja tendencia a procrastinar, mientras que puntuaciones superiores a 62 indica una alta tendencia a procrastinar (ver Tabla 3). Diversas investigaciones han encontrado que la escala tiene una estructura de factor único (Ferrari et al., 2005; Díaz-Morales et al., 2006a), aunque en un estudio italiano se han identificado recientemente dos factores (Mariani \& Ferrari, 2012).

Estas tres escalas de evaluación han sido muy utilizadas en los estudios sobre procrastinación, pero hasta el trabajo de Díaz-Morales et al. (2006a) no se había analizado su estructura factorial. A partir de este primer estudio psicométrico de las escalas de procrastinación en población española, se generó una línea de investigación para analizar si cada escala evaluaba una única dimensión o podían diferenciarse más 
Tabla 3. Procrastinación general (General Procrastination, GP; Lay, 1986)

Ítens

1. Frecuentemente me doy cuenta que estoy haciendo tareas que me había propuesto hacer con anterioridad

2. A menudo me pierdo conciertos, sucesos deportivos o lo que me gusta porque no he conseguido comprar las entradas a tiempo

3r. Cuando planeo una fiesta hago los preparativos necesarios con suficiente antelación..

4r. Cuando es hora de levantarse por la mañana, la mayoría de las veces lo hago inmediatamente

5. Puedo dejar una carta sin enviar varios días después de haberla escrito

$\begin{array}{lllll}1 & 2 & 3 & 4 & 5\end{array}$

6r. Devuelvo las llamadas de teléfono con prontitud

7. Tardo varios días en realizar trabajos, incluso los que sólo requieren sentarse y hacerlos

2345

$\begin{array}{lllll}1 & 2 & 3 & 4 & 5\end{array}$

$\begin{array}{lllll}1 & 2 & 3 & 4 & 5\end{array}$

8r. Suelo tomar decisiones lo más pronto posible

$\begin{array}{lllll}1 & 2 & 3 & 4 & 5\end{array}$

9. Generalmente me demoro en comenzar el trabajo que tengo que hacer

10. Cuando viajo, tengo que darme prisa para llegar al aeropuerto o estación a una hora apropiada

11. Cuando me preparo para salir, rara vez tengo que hacer algo en el último momento

$\begin{array}{lllll}1 & 2 & 3 & 4 & 5\end{array}$

12. Cuando estoy haciendo un trabajo que debo presentar, con frecuencia pierdo tiempo haciendo

122345

otras cosas

13r. Si llega una cuenta por una cantidad pequeña, la pago inmediatamente

12345

14r. Devuelvo pronto una petición donde se ruega una contestación rápida después de recibirla

123345

15r. Tengo una tarea acabada antes de lo necesario

122345

122345

16. Siempre parece que acabo haciendo las compras de los regalos de cumpleaños en el último minuto

12345

17. Usualmente compro, incluso lo esencial, en el último momento

123345

18r. Suelo terminar todas las cosas que planeo hacer en el día

$\begin{array}{lllll}1 & 2 & 3 & 4 & 5 \\ 1 & 2 & 3 & 4 & 5\end{array}$

19. Estoy continuamente diciendo: "lo haré mañana"

20r. Suelo ocuparme de todas las cosas que tengo que hacer antes de sentarme y relajarme por la noche

123345

12345

Nota. Para calcular su puntuación en la escala tiene que invertir la puntuación de algunas preguntas (r) y después sumarlas: a1,a2,a3r,a4r,a5,a6r,a7,a8r,a9,a10,a11r,a12,a13r,a14r,a15r,a16,a17,a18r,a19,a20r

de un componente. Así, en población universitaria británica se analizó las propiedades psicométricas de las escalas de procrastinación, planteándose si cada una de ellas podría ser una medida de la procrastinación por evitación, por indecisión o por activación (Steel, 2010). Estos sub-tipos de procrastinación serán comentarán posteriormente en esta revisión. También en población italiana (Mariani \& Ferrari, 2012), británica (Simpson \& Pychyl, 2009), turca (Ozer, Demir, \& Ferrari, 2009) y polaca (Blachnio, Przepiorka, \& DíazMorales, 2016) se han analizado psicométricamente las escalas, lo que está contribuyendo al análisis y validación del constructo procrastinación y sus posibles subdimensiones.

\section{¿Cuántas personas procrastinan?}

La investigación en los últimos 25 años ha demostrado que la procrastinación es un problema común de la sociedad occidental y refleja una tendencia muy extendida. Todas las personas posponen el inicio y/o finalización de tareas, pero no todas podrían considerase que son procrastinadoras (Ferrari, 2010). Esta aseveración constituye una llamada de atención sobre la diferencia entre retrasar una acción, algo que todas las personas realizan en alguna ocasión, y la tendencia a posponer de manera habitual el inicio y/o finalización de tareas. Si una persona demora una actividad para reunir más información o pospone una decisión porque necesita hacer algo importante antes del acto objetivo, entonces estas estrategias no constituyen acciones relacionadas con la procrastinación. No puede denominarse "procrastinación activa" al aplazamiento o postergación de actividades adecuadamente planificadas para realizar otras. Por definición, la procrastinación es un concepto que incorpora dos elementos centrales en todas sus definiciones: demora temporal y malestar subjetivo.

La procrastinación afecta prácticamente a todas las personas en mayor o menor medida. Implica saber que se debe realizar una tarea, pero que intencionadamente no se genera la motivación necesaria para realizarla dentro del intervalo de tiempo deseado (Ackerman \& Gross, 2005). Cuando la procrastinación es un comportamiento crónico, se convierte en un estilo de vida inadaptado. Se estima que el $15 \%$ de los hombres $\mathrm{y}$ mujeres adultos (entendiendo por adultos aquellas personas que participan en estudios realizados con población no universitaria y mayores de 18 años), pueden ser considerados 
como procrastinadores crónicos, personas que se involucran en un retraso innecesario de tareas relevantes en diferentes situaciones y entornos. Dos estudios transculturales han mostrado que en Australia, Perú, España, Reino Unido, Estados Unidos y Venezuela, la prevalencia de procrastinación crónica en población adulta oscila entre el $10.9 \%$ y el $16.1 \%$ (Ferrari, Díaz-Morales, O'Callaghan, Díaz, \& Argumendo, 2007).

Tabla 4. Porcentaje de personas adultas con tendencia crónica a la procrastinación

\begin{tabular}{lll}
\hline & GP & AIP \\
\hline España & 13.1 & 14.6 \\
Perú & 12.4 & 14.9 \\
Venezuela & 15.6 & 15.2 \\
Australia & 14.4 & 15.3 \\
Gran Bretaña & 10.9 & 13.8 \\
Estados Unidos & 16.1 & 15.5 \\
Total & 13.5 & 14.6 \\
\hline Nota. GP = General Procrastination; AIP = Adult Inventory \\
of Procrastination; (adaptado de Ferrari, et al., 2007).
\end{tabular}

La tendencia a procrastinar es común entre adultos que viven en estos seis países. El análisis de la prevalencia se realizó analizando las mutua relación entre la escala GP y AIP; posteriormente se desgajó la varianza específica de cada escala (utilizando la varianza residual) de la varianza común compartida entre ambas escalas, para finalmente obtener el porcentaje de aquellas personas más claramente procrastinadoras. El procedimiento puede consultarse en Ferrari et al. (2007).

\section{¿Una cuestión de sexo, edad y ocupación?}

Muchos de los estudios realizados en población universitaria indican que la prevalencia de procrastinación no difiere entre hombres y mujeres. No obstante en el meta-análisis de Van Eerde (2003) apareció una mayor tendencia a la procrastinación entre varones. Recientemente, las variables sexo/género se han tenido en cuenta en varios estudios con población adulta y sería interesante investigar cómo las tareas diarias o cotidianas asociadas a uno de los dos géneros están relacionadas con la postergación (Özer, Demir, \& Ferrari, 2009).

Con respecto a la ocupación, tampoco aparecen grandes diferencias en la tendencia a procrastinar, aunque un análisis detallado por categoría profesional indica que los empleados con nivel de cualificación profesional tienden a demostrar niveles más altos de procrastinación que empleados no cualificados (Díaz-Morales, Ferrari, Díaz, \& Argumedo, 2006b).

Finalmente, con respecto a la edad, muchos de los estudios realizados con población universitaria indicaron que no había diferencias según edad, pero el rango de edad de la población universitaria es algo restringido. Estudios con población adulta indican una disminución paulatina de la procrastinación con la edad (DíazMorales, Cohen, \& Ferrari, 2008).

\section{Componentes de la procrastinación}

Como se ha indicado anteriormente, la procrastinación se ha considerado como un constructo unidimensional pero no se analizó psicométricamente el número de factores o dimensiones que aparecen en las tres escalas de evaluación de la procrastinación más utilizadas hasta el trabajo de Díaz-Morales et al. (2016). De esta manera, sólo recientemente, la procrastinación ha comenzado a analizarse desde un punto de vista multidimensional. La lógica era analizar si la procrastinación es un constructo único, o pueden diferenciarse distintos componentes. Esta cuestión ha sido también analizada por otros autores en población italiana (Mariani \& Ferrari, 2012), británica (Simpson \& Pychyl, 2009), turca (Özer, Demir, \& Ferrari, 2009) y polaca (Blachnio, Przepiorka, \& DíazMorales, 2016).

Schouwenburg et al. (2004) consideran la procrastinación como un concepto multidimensional que aglutina una serie de componentes tales como: bajo control de impulsos, falta de persistencia, falta de disciplina en el trabajo, falta de habilidad para manejar el tiempo e incapacidad para trabajar metódicamente. Esta concepción multidimensional de la procrastinación suscitó el inicio de diversos estudios psicométricos dirigidos a analizar si pueden identificarse distintos componentes de la procrastinación o realmente la procrastinación es un constructo unitario.

En el estudio psicométrico de Díaz-Morales et al. (2006a) se analizó, por primera vez, la estructura factorial de cada escala de procrastinación 


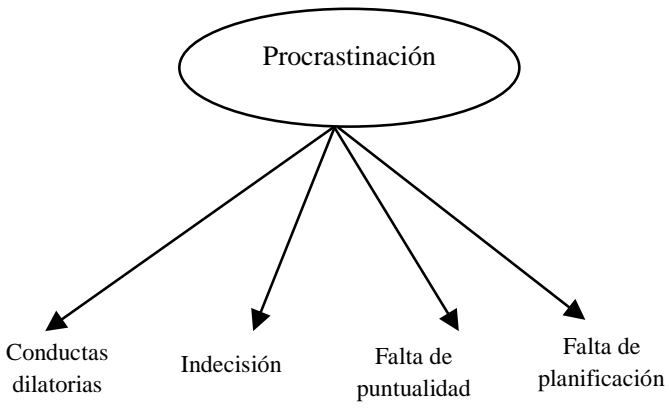

Figura 1. Esquema de la estructura factorial de las escalas GP, AIP y DP (Díaz-Morales et al., 2006a)

por separado y conjuntamente. Cuando la estructura factorial de las tres escalas combinadas se realizó en población adulta española, se identificaron cuatro componentes (ver Figura 1). Estos componentes pueden reflejar cuatro elementos esenciales de la procrastinación que pueden ser relevantes conceptualmente: conductas dilatorias, aplazamiento de la realización de la conducta que se tiene la intención de realizar (intention-behavior gap); indecisión, aplazamiento de decisiones dentro de un marco de tiempo específico; falta de puntualidad, como la incapacidad de trabajar diligentemente en una tarea para cumplir con su fecha límite; y falta de planificación, falta de autodisciplina para mantenerse enfocado en una tarea específica. El componente principal, las conductas dilatorias, configuraría el componente más "puro" de la procrastinación que aglutina el mayor porcentaje de varianza compartida entre las tres escalas de procrastinación (Díaz-Morales et al., 2006a).

Posteriormente, varios estudios han replicado esta estrategia de análisis de los componentes de la procrastinación en distintos países. Simpson y Pychyl (2009) reprodujeron un estudio psicométrico similar con estas tres escalas entre población inglesa y encontró tres factores similares: procrastinación, urgencia $y$ mantenimiento de citas, y la prontitud y realización inmediata de tareas. Mariani y Ferrari (2012) probaron diferentes modelos estructurales usando las mismas escalas de procrastinación.

En población adulta polaca (Blachnio, Przepiorka, \& Díaz-Morales, 2016), se analizó a partir de las tres escalas anteriormente mencionadas qué modelo de la procrastinación era más adecuado: un modelo unidimensional, el modelo de cuatro factores (Díaz-Morales et al., 2006a) o el modelo de tres factores (Simpson \& Pychyl, 2009). El modelo unidimensional no mostró un ajuste satisfactorio, siendo el modelo de cuatro factores sensiblemente mejor que el modelo de tres factores.

En resumen, aunque la mayor parte de la investigación realizada ha considerado que la procrastinación es un concepto unidimensional, probablemente puedan establecerse subdimensiones o componentes más específicos. La investigación dilucidará esta cuestión a partir de los datos empíricos.

\section{Modos de procrastinar}

\section{Generalizada y situacional}

Suele pensarse que la procrastinación no refleja más que una tendencia conductual común, sin consecuencias para la vida cotidiana. Ello es debido a que una persona puede posponer tareas o actividades en un área de su vida, pero no en otras áreas. Un ejemplo de procrastinación situacional es la ya mencionada procrastinación académica. Con la procrastinación académica, los estudiantes tardan en estudiar o completar tareas académicas, pero no demoran actividades de interacción social como quedar con amigos o sacar las entradas para un concierto (Solomon \& Rothblum, 1984). Se ha estimado que la procrastinación académica prevalece en aproximadamente el $70 \%$ de los estudiantes universitarios en tareas académicas como estudiar, inscribirse en clases de formularios, completar tareas de lectura o asistir a tutorías con profesores/as. Aunque las consecuencias de la procrastinación académica pueden suponer graves consecuencias para el logro educativo, estas consecuencias también son graves si la procrastinación es generalizada, o lo que es lo mismo, se convierte en un estilo de comportamiento habitual en todas las áreas vitales.

\section{Indecisión, evitación y activación}

Ferrari (1992) propuso que se podrían establecer tres tipos o modos de procrastinar debidos a la indecisión, a la evitación y a la búsqueda de activación. La procrastinación por indecisión englobaría los procesos cognitivos relacionados con el retraso voluntario del inicio y/o finalización de tareas. La indecisión puede 
llevar a los procrastinadores a crear excusas para justificar por qué no se centran en la realización de una tarea ("me surgió un asunto familiar importante que tuve que atender"), conductas de auto-sabotaje ("es que el ordenador no funciona bien, y se va a estropear") así como conductas de infravaloración del self ("es que no creo que pueda ser capaz de hacerlo"). Este nivel cognitivo de la procrastinatión también se ha relacionado con una tendencia a centrarse en el pasado negativo, rememorando las situaciones $\mathrm{y}$ conductas que tuvieron consecuencias negativas en el pasado.

Un segundo tipo de procrastinación se basa en la evitación. Por lo general, las personas posponen las cosas para evitar ciertos resultados y situaciones (Haghbin, McCaffrey, \& Pychyl, 2009). Suele ser una tendencia relacionada con una tendencia a acomodarse a las situaciones creadas por terceras personas sin mostrar una actitud activa en el logro de las tareas.

El tercer tipo de procrastinación se basaría en la búsqueda de activación o excitación, pensando que la tarea puede realizarse en el último momento, generando una falsa sensación de control y emoción por el mero hecho de retrasarla. La persona cre que puede esperar hasta el último minuto y realizar la tarea "en tiempo", aunque la investigación ha demostrado que están equivocados en su creencia de que el trabajo es mejor bajo presión (Ferrari, 2001). Investigaciones recientes, sin embargo, propusieron que la experiencia de emoción positiva reportada por algunos procrastinadores por esperar en realidad puede ser la ansiedad del estado mal etiquetada para evitar enfrentar el retraso (Simpson \& Pychyl, 2009).

\section{Estilo de personalidad del procrastinador/a}

La procrastinación se ha relacionado con una baja puntuación en el Factor Responsabilidad del Modelo de las Cinco Grandes. De hecho, para muchos autores la procrastinación puede ser el anverso de la responsabilidad. No obstante, esta relación, aunque muy relevante, nos proporciona poca información sobre el estilo de personalidad de las personas que procrastinan.

La procrastinación puede ser percibida como un mecanismo de afrontamiento no adaptativo que trata de proteger la propia autoestima cuando la persona se enfrenta a tareas exigentes así valoradas por el propio procrastinador/a. El hecho de posponer el inicio y/o finalización de la tarea, a través de las más inverosímiles excusas, produce que la persona evite las posibles valoraciones externas sobre el modo de realizar la tarea, o sobre la supuesta falta de habilidad para realizarla. De este modo, es frecuente que las personas que procrastinan se impliquen en actividades intrascendentes y momentáneas como una manera de auto-sabotearse a sí mismos/as en la realización de la tarea principal.

Un enfoque interesante para determinar el estilo de personalidad del procrastinador/a es estudiar qué motiva su conducta (metas motivacionales), cómo procesa la información (estilos cognitivos) y qué relaciones interpersonales establece (estilos interpersonales). Estos tres elementos forman el estilo de personalidad y se derivan de la teoría de personalidad de T. Millon (1990). La configuración de estas tres áreas generales, y las escalas que lo componen, configuraría el estilo de personalidad. El modelo de T. Millon es un modelo integrador muy conocido en psicología clínica puesto que constituye la base de la clasificación diagnóstica de los trastornos de personalidad del Diagnostic and Statistical Manual of Mental Disorders (DSM), pero muy poco conocido en psicología de las diferencias individuales y la psicología de la personalidad. El modelo destaca por su perspectiva integradora y comprehensiva de la personalidad, entendiendo que la personalidad estaría constituida por aquellos rasgos que han sido adaptativos en el proceso de evolución de la especie humana, y al mismo tiempo, por los modos con los que personas reaccionan a su entorno más inmediato. En este sentido, el modelo personológico de $\mathrm{T}$. Millon recoge aquellos rasgos de personalidad comunes a los diferentes modelos de personalidad planteados a lo largo del siglo XX, dotándolos de sentido en un modelo comprehensivo en donde las metas motivacionales (qué guía la conducta), los modos cognitivos (cómo se procesa la información del entorno) y las relaciones interpersonales (cómo nos relacionamos con los demás) constituyen el estilo de personalidad (Sánchez-López \& Casullo, 2000). 
Tabla 5. Estilo de personalidad relacionado con la procrastinación

\begin{tabular}{|c|c|c|c|}
\hline $\begin{array}{l}\text { Áreas y } \\
\text { escalas del MIPS }\end{array}$ & $\begin{array}{c}\text { Constructo psicológico } \\
\text { subyacente }\end{array}$ & & \\
\hline \multicolumn{4}{|l|}{ Metas motivacionales } \\
\hline S1A: Expansión & Placer & & \\
\hline S1B: Preservación & Dolor & & \\
\hline S2A: Modificación & Actividad & Baja procrastinación & \\
\hline S2B: Adecuación & Pasividad & & Alta procrastinación \\
\hline S3A: Individualidad & Uno mismo & & \\
\hline S3B: Protección & Los demás & & \\
\hline \multicolumn{4}{|l|}{ Modos cognitivos } \\
\hline S4A: Extroversión & Exterioridad & & \\
\hline S4B: Introversión & Interioridad & & \\
\hline S5A: Sensación & Tangibilidad & Baja procrastinación & \\
\hline S5B: Intuición & Intangibilidad & & Alta procrastinación \\
\hline S6A: Pensamiento & Intelecto & Baja procrastinación & \\
\hline S6B: Sentimiento & Afecto & & Alta procrastinación \\
\hline S7A.Sistematización & Asimilación & & \\
\hline S7B: Innovación & Imaginación & & \\
\hline \multicolumn{4}{|c|}{ Relaciones interpersonales } \\
\hline S8A: Retraimiento & Indiferencia & & \\
\hline S8B: Sociabilidad & Gregarismo & & \\
\hline S9A: Indecisión & Inseguridad & & Alta procrastinación \\
\hline S9B: Decisión & Confianza & Baja procrastinación & \\
\hline S10A: Discrepancia & Originalidad & & Alta procrastinación \\
\hline S10B: Conformismo & Acatamiento & Baja procrastinación & \\
\hline S11A: Sumisión & Sumisión & & \\
\hline S11B: Dominio & Dominación & & \\
\hline S12A: Descontento & Descontento & & \\
\hline S12B: Aquiescencia & Afinidad & & \\
\hline
\end{tabular}

En el estudio de Díaz-Morales, Ferrari y Cohen (2008) se analizó la relación entre la procrastinación (medida por el cuestionario Adult Inventory Procrastination, AIP) y los estilos de personalidad (medida por el Inventario Millon de Estilos de Personalidad, MIPS; Millon, 2001). Los resultados indicaron que una alta puntuación en procrastinación se asociaba con la escala Adecuación del área de Metas Motivacionales, indicando que las personas con una tendencia a procrastinar hacen poco por dar forma a sus vidas o modificarlas. Ante los acontecimientos, reaccionan adaptándose a las circunstancias que otros crean. Parecen dar su consentimiento sin protestar, son incapaces de volverse activos, carecen de iniciativa y hacen muy poco para provocar los resultados que desean. Constituiría una tendencia a la inacción o passividad y acomodamiento a circunstancias creadas por otro (véase Tabla 5).

En segundo lugar, como estilo cognitivo, las personas procrastinadoras prefieren aprender a partir de información intangible e intuiciones, con preferencia por lo simbólico y lo desconocido a lo concreto y lo observable (escala Intangibilidad). Además, una vez que han adquirido la información del entorno a partir de lo simbólico y lo ambiguo, organizan la información usando parámetros emocionales y subjetivos (escala Sentimiento). En este sentido, prefieren aprender partir de información simbólica y organizar lo aprendido a partir de su propia afectividad.

Por último, dentro del área de las relaciones interpersonales, las personas con alta tendencia a procrastinar destacan por su indecisión a la hora 
de relacionarse con los demás, generalmente se muestran tímidas y nerviosas en contextos sociales, deseando fervientemente gustar y ser aceptados por los demás, aunque con frecuencia temen ser rechazados (escala Indecisión). En segundo lugar, destacan por su tendencia a discrepar de los convencionalismos y costumbres sociales, con frecuencia se resisten a seguir las normas tradicionales y despliegan una audacia que puede ser considerada imprudente o vehemente y ambiciosa (escala Discrepancia).

Atendiendo al constructo psicológico que subyace a cada escala de las reseñadas, se podría concluir que el estilo de personalidad de las personas con alta tendencia a la procrastinación estaría formado por una tendencia a la pasividad (metas motivacionales), preferencia por información simbólica que organizan a través de su afectividad (modos cogntivos), y tendencia a la indecisión y a la discrepancia respecto a las normas y costumbres sociales (relaciones interpersonales).

\section{Una cuestión de tiempo}

El tiempo físico es constante: ¡no vuela! Hay 24 horas en un día, todos los 365 días del año. El tiempo se mueve constantemente y de modo uniforme, pero la percepción de la persona es subjetiva, ni constante ni uniforme. Esto implica, muchos fenómenos relacionados con la estimación del tiempo y su uso para hacer que el tiempo "pase" más deprisa o más despacio. Cuando se trata de estimar el tiempo que llevará realizar una tarea, los procrastinadores subestiman el tiempo necesario para ello, creyendo que tienen tiempo suficiente para realizarla y aplazando su inicio y/o finalización.

La procrastinación tiene características afectivas, conductuales y cognitivas que reflejan una gestión del tiempo ineficiente (Ferrari et al., 1995), lo que implica el incumplimiento de los plazos dentro de un intervalo temporal específico. La existencia del intervalo temporal, es en sí mismo, un factor esencial de la definición de procrastinación.

Las personas que procrastinan en comparación con las que no procrastinan dedican menos tiempo de preparación en tareas que probablemente tuvieron éxito en el pasado y más tiempo en proyectos que probablemente fracasen
(Lay, 1990), subestiman el tiempo total requerido para completar una tarea, dedican menos tiempo buscando la información necesaria para completar tareas, inician tareas académicas (por ejemplo, estudiar para exámenes) en el último minuto, y están más "orientados/as al presente" (Ferrari et al., 1995). Vodanovich y Seib (1997) indicaron que las personas con una mayor tendencia a procastinar estructuran peor su tiempo y valoran el uso del tiempo menos significativamente que las personas que no procrastinan. Aunque en el contexto laboral, Van Eerde (2003) mostró que la formación en gestión del tiempo disminuía posteriormente la tendencia a procrastinar, la procrastinación no es únicamente un problema de gestión del tiempo.

\section{Perspectiva temporal: pasado, presente $\mathbf{y}$ futuro}

Debido a que la procrastinación es a veces considerada como "la mejor manera de perder el tiempo", algunos estudios se han ocupado de estudiar la relación entre la perspectiva temporal y la procrastinación. La perspectiva temporal, la capacidad de pensar en el pasado, presente y futuro, puede que sea la característica más fundamental del ser humano desde el punto de vista evolucionista (Sunddendorf \& Corballis, 1997). La perspectiva temporal se ha asociado con variables psicológicas como el grado de bienestar, optimismo, controlabilidad, autodirección, la motivación de logro y la procrastinación (Zaleski, 1996).

Los procrastinadores crónicos prefieren actividades a corto plazo, de modo que así evitan metas futuras, reduciendo la ansiedad que supone planteárselas, buscando recompensas inmediatas más que recompensas a largo plazo. De esta manera, los procrastinadores crónicos buscarán recompensas placenteras inmediatas en el momento presente.

La investigación previa ha demostrado que la procrastinación se relaciona preferentemente con la perspectiva temporal presente más que con la perspectiva temporal futura. Un énfasis en la perspectiva de tiempo futuro requiere una perspectiva a largo plazo, trazar un plan de acción y completar las actividades en un tiempo determinado. Una persona orientada al futuro puede necesitar más tiempo para tomar decisiones 
importantes, especialmente cuando el intervalo temporal es amplio en el curso de la acción, pero ello no le genera malestar y no evita la realización de actividades que conduzcan a conseguir su objetivo.

Specter y Ferrari (2000) encontraron que la procrastinación por indecisión se relacionaba con una baja perspectiva temporal futura y alta perspectiva temporal pasada; Jackson et al. (2003) encontraron que la procrastinación académica estaba relacionada con una baja orientación al futuro, alta orientación a un presente fatalista y a un pasado negativo. Claramente, en estos dos estudios donde se utilizaron dos escalas diferentes de procrastinación (procrastinación decisional y académica), la procrastinación se asoció con una baja perspectiva temporal futura. Aún así, la afirmación común en relación a que los procrastinadores prefieren recompensas placenteras inmediatas en el presente no fue confirmada.

Más recientemente, varios estudios encontraron que la procrastinación se asociaba con mayor orientación al presente fatalista y/o hedonista y con menor orientación futura (DíazMorales, Ferrari, \& Cohen, 2008).

El Inventario de Perspectiva del Tiempo de Zimbardo (ZTPI) (Díaz-Morales, 2006: Lomelí, de los Ángeles, Cárdenas-Niño, \& Tapia-Fonllem, 2018; Ortuño, Nunes Janeiro, Paixão, Esteves, \& Cordeiro, 2017) diferencia cinco orientaciones temporales, las cuales se asocian con la procrastinación.

Orientación futura: Las personas orientadas al futuro tienden a planificar y organizar cuidadosamente sus actividades laborales, lo cual es la tendencia opuesta a la procrastinación que se caracteriza por baja persistencia, disciplina, habilidades no óptimas de administración del tiempo y la baja capacidad de trabajar metódicamente (Milgram \&Tenne, 2000).

Orientación a un pasado positivo. El énfasis en la orientación del tiempo pasado puede permitir a una persona adoptar una perspectiva a largo plazo, evitar riesgos y enfatizar la estabilidad. La persona se centraría en sus logros pasados y los cursos de acción que tuvieron éxito, despertando una sensación positiva de bienestar.

Orientación pasado negativo. En este otro caso, la orientación a un pasado negativo se asocia con sentimientos de infravaloración y pesimismo. La persona se centra en sus errores y en la imposibilidad de superarlos. Las personas que procrastinan por indecisión suelen adoptar una orientación al pasado negativa, demasiado preocupados por recordar sucesos pasados negativos, y esta forma de reflexión, aparece la indecisión.

Orientación presente hedonista. Un énfasis en la orientación del tiempo presente hedonista puede facilitar que una persona viva aquí y ahora centrándose en perspectivas a corto plazo (Brislin \& Kim, 2003). Sería propia de aquellas personas que procrastinan creyendo que pueden realizar la tarea en el último momento, posponiendo el inicio y/o finalización de la tarea hasta la fecha límite. Tal estrategia puede energizar a la persona para que trabaje rápidamente, especialmente si la tarea no es atractiva o no es desafiante (Van Eerde, 2003), aunque la mayor parte de las veces se trata de un sesgo cognitivo que implica la infravaloración del tiempo necesario para acometer la tarea, y una sobrevaloración de las capacidades de uno/a mismo/a para realizarla. Los procrastinadores suelen realizar actividades placenteras, en el corto plazo, y suelen poner excusas para ganar tiempo adicional para completar tareas, lo que generalmente se asocia con sentimientos de malestar con uno/a mismo/a y con la situación (Ferrari et al., 1995).

Orientación presente fatalista: las personas con una alta orientación al presente fatalista muestran bajos niveles de escrupulosidad, tendencia a la depresión y altos niveles de inestabilidad emocional, lo que se relaciona con la evitación de tareas propia de los procrastinadores (Dewitte \& Schouwenburg, 2002).

Ferrari y Díaz-Morales (2007a) encontraron que procrastinación por evitación (evaluada mediante la escala AIP) se relacionó con una alta orientación al presente fatalista, mientras que la procrastinación por activación (evaluada mediante la escala GP) se relacionó con una alta orientación al presente hedonista y una baja orientación al futuro (Tabla 6). Tal vez, ambas escalas de procrastinación miden diferentes formas de procrastinación: más relacionadas con la evitación (AIP) y más relacionadas con la activación (GP). 
Tabla 6. Relaciones entre la perspectiva temporal y la procrastinación por evitación (AIP) y la procrastinación por activación (GP)

\begin{tabular}{lcc}
\hline $\begin{array}{l}\text { Escalas del } \\
\text { ZTPI }\end{array}$ & AIP & GP \\
\hline $\begin{array}{l}\text { Pasado } \\
\text { Negativo }\end{array}$ & \\
$\begin{array}{l}\text { Presente } \\
\text { Hedonista }\end{array}$ & $\begin{array}{c}\text { Alta procrastinación } \\
\text { por activación } \\
\text { Baja procrastinación } \\
\text { por activación }\end{array}$ \\
$\begin{array}{l}\text { Pasado } \\
\text { Positivo }\end{array}$ & \\
Presente & Procrastinación \\
Fatalista & por \\
evitación & \\
$\begin{array}{l}\text { Nota. GP = General Procrastination; AIP = Adult Inventory } \\
\text { 2007a). }\end{array}$ &
\end{tabular}

\section{Matutinidad-vespertinidad: alondras y búhos}

Otro aspecto temporal de la conducta humana tiene que ver con los ritmos circadianos, aquellos que se suceden con una periodicidad de 24 horas tales como el ciclo vigilia-sueño, la temperatura corporal o la secreción de hormonas como el cortisol y la melatonina. Además de esta periodicidad circadiana de 24 horas, existen personas matutinas cuyos ritmos de 24 horas oscilan en sincronía con el ciclo luz/oscuridad y se levantan y acuestan con la salida y el ocaso del sol. Mientras que los ritmos circadianos de las personas vespertinas suelen estar ligeramente retrasados con respecto al ciclo luz/oscuridad, lo que hace que tengan más dificultades para levantarse y acostarse pronto, las personas matutinas se levantan y acuestan más en sincronía con el ciclo luz/oscuridad.

Los resultados indican que las personas vespertinas tienden a ser más procrastinadoras. Dado que las personas vespertinas se encuentran en su mejor momento por la tarde-noche, es probable que ello les conduzca a aplazar las tareas hasta el final de día, lo que en una sociedad matutina, puede generar una valoración desfavorable por terceras personas.

En el estudio de Díaz-Morales, Ferrari y Cohen (2008) se analizaron las relaciones entre la procrastinación, la perspectiva temporal y la matutinidad-vespertinidad. Se analizó qué papel tenía la perspectiva temporal y la matutinidadvepsertinidad cuando se consideraba el modo de procrastinación por indecisión y el modo de procrastinación por evitación (o más conductualmente observable). Así, ambos tipos de procrastinación, más cognitiva (indecisión) y más conductualmente observable (evitación) se relacionarían de modo diferente con la orientación temporal y con la matutinidad-vespertinidad.

Tal y como aparece en la Tabla 7, la procrastinación por evitación se asoció con la tendencia a la vespertinidad. Las personas procrastinadoras demoran y evitan realizar sus tareas hasta el final de día, lo cual es la característica central de las personas vespertinas. La cuestión que queda abierta es si las personas vespertinas (a nivel biológico) realizan mejor sus actividades por la tarde noche y ello hace que puntúen alto en procrastinación, o a la inversa, si las personas procrastinadoras al evitar sus tareas y demorarlas hasta el final del día, resulta que puntúan alto en vespertinidad.

En segundo lugar, la procrastinación por indecisión se asocia con alta orientación al pasado positivo y negativo, así como con baja orientación al futuro y al presente hedonista.

Tabla 7. Relaciones entre la procrastinación por evitación (AIP), por indecisión (DP), la perspectiva temporal (ZTPI) y la matutinidadvespertinidad (CSM)

\begin{tabular}{|c|c|c|}
\hline $\begin{array}{l}\text { Escalas del } \\
\text { ZTPI }\end{array}$ & $\begin{array}{l}\text { Procrastinación } \\
\text { por evitación } \\
\text { (AIP) }\end{array}$ & $\begin{array}{l}\text { Procrastinación } \\
\text { por Indecisión } \\
\text { (DP) }\end{array}$ \\
\hline $\begin{array}{l}\text { Pasado } \\
\text { Negativo } \\
\text { Presente } \\
\text { Hedonista }\end{array}$ & \multirow{3}{*}{$\begin{array}{c}\text { Baja } \\
\text { orientación al } \\
\text { futuro }\end{array}$} & $\begin{array}{l}\text { Alta orientación } \\
\text { pasado negativo } \\
\text { Baja orientación } \\
\text { presente hedonista }\end{array}$ \\
\hline Futuro & & $\begin{array}{c}\text { Baja orientación al } \\
\text { futuro }\end{array}$ \\
\hline $\begin{array}{l}\text { Pasado } \\
\text { Positivo } \\
\text { Presente } \\
\text { Fatalista }\end{array}$ & & \multirow[t]{2}{*}{$\begin{array}{l}\text { Alta orientación } \\
\text { pasado positivo }\end{array}$} \\
\hline $\begin{array}{l}\text { Escala CSM } \\
\text { Matutinidad- } \\
\text { Vespertinidad } \\
\end{array}$ & $\begin{array}{c}\text { Tendencia a la } \\
\text { vespertinidad }\end{array}$ & \\
\hline $\begin{array}{l}\text { lota. ZTPI= } \\
\text { imbardo; CSN } \\
\text { lespertinidad; } \\
\text { dultos; DP = } \\
\text { daptado de Día }\end{array}$ & $\begin{array}{l}\text { tario de Per } \\
\text { Escala Comp } \\
=\text { Inventario c } \\
\text { cala de Proc } \\
\text { rales, Ferrari y }\end{array}$ & $\begin{array}{l}\text { tiva Temporal de } \\
\text { a de Matutinidad } \\
\text { rocrastinación para } \\
\text { inación Decisional } \\
\text { en (2008) }\end{array}$ \\
\hline
\end{tabular}

Posteriormente, otros estudios han encontrado resultados similares. Estudiantes polacos matutinos tienen a estar orientados al futuro, mientras que los estudiantes vespertinos lo están 
en mayor media hacia el presente (Matthews \& Stolarski, 2015).

\section{Comentarios Finales}

La procrastinación es una tendencia muy generalizada entre la población occidental, mediante la cual se demora el inicio y/o finalización de tareas que podrían realizarse en un tiempo establecido y que se acompaña de sentimientos de malestar. Como se ha indicado, no toda conducta de postergación o aplazamiento implica procrastinación. Todas las definiciones incorporan dos elementos: marco temporal y malestar subjetivo. El aplazamiento de la conducta genera un sentimiento de malestar en la persona porque no inicia y/o finaliza la tarea en el tiempo establecido. A nivel cognitivo, la persona genera todo tipo de sesgos para preservar su nivel de autoestima y no sentirse valorada por los demás; a nivel conductual, es probable que se implique en tareas que nada tienen que ver con el cumplimento de la tarea objetivo, constituyendo toda una suerte de conductas de auto-sabotaje distractoras. A nivel emocional, el sentimiento de infravaloración, ansiedad, y afectividad, genera una tendencia a la pasividad que no es energía o motiva para acometer la tarea. A nivel social, las personas que procrastinan son indecisas en sus relaciones interpersonales, pensando constantemente en la opinión que los demás pueden tener de uno/a misma, y cuestionando constantemente los modos tradicionales y establecidos de hacer las cosas. El tratamiento de la procrastinación implica realizar algo más que un curso de gestión de tiempo. Como ha quedado patente en diversas investigaciones, debe abordarse desde la esfera cognitiva, afectiva y conductual, identificando el estilo de personalidad que procrastina, y ajustando el tratamiento a sus peculiaridades. Las diferencias individuales, así pues, hacen que las recetas generales no sean eficaces ni eficientes.

\section{Referencias}

Ackerman, D. S., \& Gross, B. L. (2005). My instructor made me do it: Task characteristics of procrastination. Journal of Marketing Education, 27, 5-13. doi:https://doi.org/10.1177/0273475304273842

Aitken, M. E. (1982). A personality profile of the college student procrastinator (Doctoral dissertation, University of Pittsburgh, 1982). Dissertation Abstracts International, 43, 722.

Ajzen, I. (1991). The theory of planned behavior. Organizational Behavior and Human Decision Processes, 50, 179-211. doi:https://doi.org/10.1016/0749-5978(91)90020-T

Argumedo, D., Díaz, K., Calderón, A., DíazMorales, J. F., \& Ferrari, J.R. (2005). Evaluación de la confiabilidad y la estructura factorial de tres escalas de procrastinación crónica. Revista de Psicología de la Pontificia Universidad Católica del Perú (PUCP), XVIII, 113-138.

Ariely, D., \& Wertenbroch, K. (2002). Procrastination, deadlines, and performance: Self-control by precommitment. Psychological Science, 13,

219-224. doi:https://doi.org/10.1111/1467-9280.00441

Blachnio, A., Przepiorka, A., \& Díaz-Morales, J.F. (2016). I will do it tomorrow! Exploring the dimensionality of procrastination in Poland. Time and Society. (First published date: December-01-2016). doi:https://doi.org/10.1177/0961463X16678251

Brislin, R. W., \& Kim, E. S. (2003). Cultural diversity in people's understanding and uses of time. Applied Psychology, 52, 363-382. doi:https://doi.org/10.1111/1464-0597.00140

Choi, J. N., \& Moran, S. V. (2009). Why not procrastinate? Development and validation of a new active procrastination scale. The Journal of Social Psychology, 149, 195-212. doi:https://doi.org/10.3200/SOCP.149.2.195-212

Chun Chu, A. H., \& Choi, J. N. (2005). Rethinking procrastination: Positive effects of "active" procrastination behavior on attitudes and performance. The Journal of Social Psychology, 145, 245-264. doi:https://doi.org/10.3200/SOCP.145.3.245-264

Dewitte, S., \& Schouwenburg, H. C. (2002). Procrastination, temptations, and incentives: The struggle between the present and the future in procrastinators and the punctual. European Journal of Personality, 16, 469-489. doi:https://doi.org/10.1002/per.461

Díaz, K., Argumedo, D., \& Díaz-Morales, J.F. (2009). Procrastinación y estilos de 
personalidad (pp. 193-214). En D. Herrera (ed.). Teorías contemporáneas de la motivación: una perspectiva aplicada. Lima: Fondo Editorial de la PUCP.

Díaz-Morales, J. F. (2006). Estructura factorial y fiabilidad del Inventario de Perspectiva Temporal de Zimbardo. Psicothema, 18, 565571.

Díaz-Morales, J. F., Ferrari, J. R., \& Cohen, J.R. (2008). Indecision and avoidant procrastination: The role of morningnesseveningness and time perspective in chronic delay lifestyles. The Journal of General Psychology, 135, 228-240. doi:https://doi.org/10.3200/GENP.135.3.228-240

Díaz-Morales, J.F. \& Ferrari, J.R. (2015). More time for procrastinators: The role of time perspective (pp. 305-321). In M. Stolarski, N. Fieulaine, \& W. van Beek (eds.). Time perspective theory: Review, research and application. London: Springer. doi:https://doi.org/10.1007/978-3-319-07368-2_20

Díaz-Morales, J. F., Ferrari, J. R., \& Cohen, J. (2008). Millon's personality styles related to procrastination: A dynamic view of the personality styles of chronic task delays. Personality and Individual Differences, $\quad 45, \quad 554-$ 558. doi:https://doi.org/10.1016/j.paid.2008.06.018 Díaz-Morales, J. F., Ferrari, J. R., Díaz, K., \& Argumedo, D. (2006a). Factorial structure of three procrastination scales with Spanish adult population. European Journal of Psychological Assessment, 22, 132-137. doi:http://dx.doi.org/10.1027/1015-5759.22.2.132

Díaz-Morales, J. F., Ferrari, J. R., Díaz, K., \& Argumedo, D. (2006b). Procrastination and demographic characteristics in Spanish adults: Further evidence. The Journal of Social Psychology, 146, 629-633. doi:https://doi.org/10.3200/SOCP.146.5.629-633

Digdon, N. L., \& Howell, A. J. (2008). College students who have an eveningness preference report lower self-control and greater procrastination. Chronobiology International, 25, 1029-1046.

doi:https://doi.org/10.1080/07420520802553671

EPSY. Estilos de personalidad, género y salud. Grupo de Investigación Consolidado de la
Universidad Complutense de Madrid. https://www.ucm.es/epsy/

Ericson, K. M. (2017). On the interaction of memory and procrastination: Implications for reminders, deadlines, and empirical estimation. Journal of the European Economic Association, 15, 692-719. doi:https://doi.org/10.1093/jeea/jvw015

Ferrari, J. R. (1992). Psychometric validation of two procrastination inventories for adults: Arousal and avoidance measures. Journal of Psychopathology and Behavioral Assessment, 14, 97-110. doi:https://doi.org/10.1007/BF00965170

Ferrari, J. R. (2001). Procrastination as selfregulation failure of performance: Effects of cognitive load, self-awareness, and time limits on 'working best under pressure'. European Journal of Personality, 15, 391-406. doi:https://doi.org/10.1002/per.413

Ferrari, J. R., \& Pychyl, T. A. (2000). Procrastination: Current issues and new directions. Corte Madre, CA: Select Press.

Ferrari, J. R., Johnson, J. L., \& McCown, W. G. (1995). Procrastination and Task Avoidance. Springer US.

Ferrari, J. R., Özer, B. U., \& Demir, A. (2009). Chronic procrastination among Turkish adults: Exploring decisional, avoidant, and arousal styles. The Journal of Social Psychology, 149, 402-408. doi:https://doi.org/10.3200/SOCP.149.3.402-408

Ferrari, J. R., \& Díaz-Morales, J. F. (2007b). Perceptions of self-concept and selfpresentation by procrastinators: Further evidence. The Spanish Journal of Psychology, 10, 91-96.

https://doi.org/10.1017/S113874160000634X

Ferrari, J. R., \& Díaz-Morales, J. F. (2007a). Procrastination: Different time orientations reflect different motives. Journal of Research in Personality, 41, 707-714. doi:https://doi.org/10.1016/j.jrp.2006.06.006

Ferrari, J. R. (2010). Still procrastinating: The no regrets guide to getting it done. New Jersey: John Wiley \& Sons.

Ferrari, J. R., Díaz-Morales, J. F., O'Callaghan, J., Díaz, K., \& Argumedo, D. (2007). Frequent behavioral delay tendencies by adults: international prevalence 
rates of chronic procrastination. Journal of Cross-Cultural Psychology, 38, 458-464. doi:https://doi.org/10.1177/0022022107302314

Ferrari, J. R., Driscoll, M., \& Díaz-Morales, J. F. (2007). Examining the self of chronic procrastinators: Actual, ought, and undesired attributes. Individual Differences Research, 5, 115-123.

Haghbin, M., McCaffrey, A., \& Pychyl, T. A. (2012). The complexity of the relation between fear of failure and procrastination. Journal of Rational-Emotive \& Cognitive-Behavior Therapy, 30, 249-263. https://doi.org/10.1007/s10942-012-0153-9

Jackson, T., Fritch, A., Nagasaka, T., \& Pope, L. (2003). Procrastination and perceptions of past, present, and future. Individual Differences Research, 1, 17-28.

Lay, C. H. (1986). At last, my research article on procrastination. Journal of Research in Personality, 20, 474-495. doi:https://doi.org/10.1016/0092-6566(86)90127-3

Lay, C. H. (1990). Working to schedule on personal projects: An assessment of personproject characteristics and trait procrastination. Journal of Social Behavior and Personality, 5, 91-103.

Lomelí, D. G., de los Ángeles Maytorena, M., Cárdenas-Niño, L., \& Tapia-Fonllem, C. O. (2018). Perspectiva temporal de estudiantes universitarios Mexicanos y Colombianos. Revista Iberoamericana de Diagnóstico y Evaluación - e Avaliação Psicológica, 46, 133-145. doi:https://doi.org/10.21865/RIDEP46.1.10

Mann, L. (1982). Decision Making Questionnaire: Parts I and II. In J. R. Ferrari, J. L. Johnson, \& W. McCown (Eds.), Procrastination and task avoidance. New York: Plenum Press.

Mariani, M. G., \& Ferrari, J. R. (2012). Adult Inventory of Procrastination scale (AIP): A comparison of model with and Italian sample. Testing, Psychometric, Methodology in Applied Psychology, 19, 3-14.

Matthews, G., \& Stolarski, M. (2015). Emotional processes in development and dynamics of individual time perspective. In M. Stolarski, N. Fieulaine, \& W. van Beek (eds.). Time perspective theory: Review, research and application. London: Springer. doi:https://doi.org/10.1007/978-3-319-07368-2_18
McCown, W., Johnson, J., \& Petzel, T. (1989). Procrastination, a principal components analysis. Personality and Individual Differences, 10, 197-202. doi:https://doi.org/10.1016/0191-8869(89)90204-3

Metin, U. B., Taris, T. W., \& Peeters, M. C. (2016). Measuring procrastination at work and its associated workplace aspects. Personality and Individual Differences, 101, 254-263. doi:https://doi.org/10.1016/j.paid.2016.06.006

Millon, T. (1990). Toward a new personology: An evolutionary model. John Wiley \& Sons.

Millon, T. (2001) Inventario Millon de Estilos de Personalidad (MIPS). Madrid: TEA Ediciones.

Ortuño, V. E., Nunes Janeiro, I., Paixão, M. P., Esteves, C., \& Cordeiro, P. (2017). Um novo modelo multidimensional da Perspetiva Temporal. In Revista Iberoamericana de Diagnóstico y Evaluación - e Avaliação Psicológica, $\quad 45, \quad 71-84$. doi:https://doi.org/10.21865/RIDEP45.3.06

Özer, B. U., Demir, A., \& Ferrari, J. R. (2009). Exploring academic procrastination among Turkish students: possible gender differences in prevalence and reasons. The Journal of Social Psychology, 149, 241-257. doi:https://doi.org/10.3200/SOCP.149.2.241-257

Przepiorka, A., Błachnio, A., \& Díaz-Morales, J.F. (2016). Problematic Facebook use and procrastination. Computers in Human Behavior, 65, 59-64. doi:https://doi.org/10.1016/j.chb.2016.08.022

Sánchez-López, M. P., \& Casullo, M. M. (2000) (eds.). Estilos de personalidad: Una perspectiva iberoamericana. Madrid: Miño y Dávila.

Schouwenburg, H. C., \& Lay, C. H. (1995). Trait procrastination and the big-five factors of personality. Personality and Individual Differences, $\quad 18, \quad 481-490$. doi:https://doi.org/10.1016/0191-8869(94)00176-S

Schouwenburg, H. C., Lay, C. H., Pychyl, T. A., \& Ferrari, J. R. (2004). Counseling the procrastinator in academic settings. Washington, DC: American Psychological Association.

Simpson, W. K., \& Pychyl, T. A. (2009). In search of the arousal procrastinator: Investigating the relation between 
procrastination, arousal-based personality traits and beliefs about procrastination motivations. Personality and Individual Differences, 47(8), 906-911. doi:https://doi.org/10.1016/j.paid.2009.07.013

Soysa, C. K., \& Weiss, A. (2014). Mediating perceived parenting styles-test anxiety relationships: Academic procrastination and maladaptive perfectionism. Learning and Individual Differences, 34, 77-85. doi:https://doi.org/10.1016/j.lindif.2014.05.004

Solomon, L. J., \& Rothblum, E. D. (1984). Academic procrastination: Frequency and cognitive-behavioral correlates. Journal of Counseling Psychology, 31, 503-509. http://dx.doi.org/10.1037/0022-0167.31.4.503

Specter, M. H., \& Ferrari, J. R. (2000). Time orientations of procrastinators: Focusing on the past, present, or future?. Journal of Social Behavior and Personality, 15, 197-202.

Steel, P. (2010). Arousal, avoidant and decisional procrastinators: Do they exist? Personality and Individual Differences, 48, 926-934. doi:https://doi.org/10.1016/j.paid.2010.02.025

Steel, P. (2007). The nature of procrastination: A meta-analytic and theoretical review of quintessential self-regulatory failure. Psychological Bulletin, 133, 65-94. doi:http://dx.doi.org/10.1037/0033-2909.133.1.65

Steel, P., \& Ferrari, J. (2013). Sex, education and procrastination: an epidemiological study of procrastinators' characteristics from a global sample. European Journal of Personality, 27, 51-58. doi:https://doi.org/10.1002/per.1851

Steel, P., Svartdal, F., Thundiyil, T., \& Brothen, T. (2018). Examining procrastination across multiple goal stages: A longitudinal study of temporal motivation theory. Frontiers in $\begin{array}{lll}\text { Psychology, } & 927 .\end{array}$ doi:https://doi.org/10.3389/fpsyg.2018.00327

Suddendorf, T., \& Corballis, M. C. (1997). Mental time travel and the evolution of the human mind. Genetic, Social, and General Psychology Monographs, 123, 133-167.

Milgram, N., \& Tenne, R. (2000). Personality correlates of decisional and task avoidant procrastination. European Journal of Personality, 14, 141-156. doi:https://doi.org/10.1002/(SICI)1099-
0984(200003/04)14:2<141::AID-

PER369>3.0.CO;2-V

Tuckman, B. W. (1991). The development and concurrent validity of the procrastination scale. Educational and Psychological Measurement, $\quad 51, \quad$ 473-480. doi:https://doi.org/10.1177/0013164491512022

Uzun, B., Ferrari, J. R., \& LeBlanc, S. (2018). Put aside procrastination: Positive emotional outcomes from self-forgiveness for delays. North American Journal of Psychology, 20, 171-186.

Van Eerde, W. (2003). A meta-analytically derived nomological network of procrastination. Personality and Individual Differences, $\quad 35, \quad$ 1401-1418. doi:https://doi.org/10.1016/S0191-8869(02)00358-6

Vodanovich, S. J., \& Seib, H. M. (1997). Relationship between time structure and procrastination. Psychological Reports, 80, 211-215. doi:https://doi.org/10.2466/pr0.1997.80.1.211

Zaleski, Z. (1996). Future anxiety: Concept, measurement, and preliminary research. Personality and Individual Differences, 21, 165-174. doi:https://doi.org/10.1016/0191-8869(96)00070-0 